\title{
MXene材料用于放射性元素及重金属离子去除的 研究进展
}

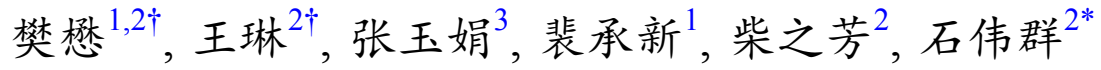 \\ 1 国民核生化灾害防护国家重点实验室, 北京 102205 \\ 2 中国科学院高能物理研究所, 北京 100049 \\ 3 北京科技大学材料科学与工程学院, 北京 100083 \\ †同等贡献 \\ *通讯作者, E-mail: shiwq@ihep.ac.cn \\ 收稿日期: 2018-06-27; 接受日期: 2018-08-28; 网络版发表日期: 2018-12-25 \\ 国家自然科学基金(编号: 21577144, 11675192, 21790373)资助项目
}

摘要 二维过渡金属碳/氮化物(MXene)是2011年发现的一类结构新颖、性质独特的层状纳米材料, 目前已被 广泛应用于能源存储以及环境治理等领域. MXene材料拥有较大的比表面积、丰富的活性吸附位点、良好的亲 水性以及可控的层间距, 并且这类纯无机材料表现出优异的抗辐照性能以及良好的导热性能, 因此是容纳放射性 元素与重金属离子的理想载体。本文归纳总结了近年来MXene材料的可控制备及其对放射性元素和重金属离子 的去除研究进展, 对相关的实验技术手段、吸附行为与相互作用机理进行了系统梳理, 此外还对该领域的发展趋 势进行了展望.

关键词 MXene, 放射性核素, 重金属离子, 吸附, 环境修复

\section{1 引言}

核能是一种能量密度高、技术成熟的清洁能源, 提供了全世界 $11 \%$ 的电力，是未来能源发展战略的重 点 ${ }^{[1]}$. 伴随着核能发展规模的提升, 铀矿开采、核废物 处理与处置等核燃料循环相关的活动频次不断增强, 天然或人为因素导致的放射性核素泄漏几率以及由此 形成的放射性污染风险也显著增加. 常见的放射性核 素(如 ${ }^{235} \mathrm{U} 、{ }^{238} \mathrm{U} 、{ }^{239} \mathrm{Pu} 、{ }^{241} \mathrm{Am} 、{ }^{90} \mathrm{Sr} 、{ }^{137} \mathrm{Cs} 、{ }^{131} \mathrm{I}$ 和 ${ }^{99} \mathrm{Tc}$ ) 通常具有较强的辐射损伤效应(放射性)、化学
毒性以及环境迁移能力，一旦泄露会对土壤、水资源 以及生态环境造成长期危害 ${ }^{[2-4]}$. 另一方面, 近年来采 矿、冶金、化工以及电子加工等行业的快速发展产生 了大量含有重金属离子的工业废水，其中典型的代表 性重金属阳离子 $\left(\mathrm{Hg}^{2+} 、 \mathrm{~Pb}^{2+} 、 \mathrm{Cd}^{2+} 、 \mathrm{Cu}^{2+}\right.$ 等 $)$ 以及含 氧阴离子 $\left(\mathrm{CrO}_{4}{ }^{2-} 、 \mathrm{Cr}_{2} \mathrm{O}_{7}{ }^{2-} 、 \mathrm{AsO}_{4}{ }^{3-}\right.$ 等 $)$ 可使水体和土 壤环境恶化, 并能够通过食物链以及直接接触等方式 危及人类社会和公众健康 ${ }^{[5]}$. 相较于其他有机污染物, 放射性元素和有毒重金属离子无法降解为环境友好的 产物, 并且它们造成的环境污染具有浓度低、面积

引用格式: Fan M, Wang L, Zhang Y, Pei C, Chai Z, Shi W. Research progress of MXene materials in radioactive element and heavy metal ion sequestration. Sci Sin Chim, 2019, 49: 27-38, doi: 10.1360/N032018-00155 
大、易迁移、持续累积性、隐蔽性以及不可逆性等特 点，因此高效去除放射性元素与重金属离子污染极具 挑战性，目前已成为国际上环境治理研究领域的难点 与热点.

近年来，科研工作者制备了一系列功能性的固相 吸附剂材料用于放射性元素与重金属离子的高效富集 与分离回收基础研究. 其中, 以功能化石墨烯 ${ }^{[6]}$ 、金属 有机框架材料 ${ }^{[7]}$ 、纳米多孔高分子聚合物 ${ }^{[8]}$ 等为代表 的有机类材料具有吸附容量高、选择性好等优点，但 该类材料的化学与结构稳定性较差，尤其对于放射性 核素分离可能存在的高酸度、强辐照场、高释热等极 端条件无法适用; 而以沸石 ${ }^{[9]}$ 、天然黏土矿物 ${ }^{[10]}$ 、金 属氧化物/氢氧化物纳米材料 ${ }^{[1]}$ 为代表的无机类材料 具有稳定性好、制备简单、成本低廉等特点，但受限 于较少的活性位点，它们的高效富集分离性能有所欠 缺. 因此, 针对性地开发出吸附容量高、选择性好, 同 时兼具良好稳定性的新型无机纳米材料是高效实用去 除放射性核素和有毒重金属离子的重点研究方向.

MXene材料包括层状过渡金属碳化物、碳氮化物 以及氮化物，具有二维六方结构以及 $\mathrm{M}_{n+1} \mathrm{X}_{n}$ 的化学通 式，主要由层状三元金属陶瓷材料(MAX相，通式 $\mathrm{M}_{n+1} \mathrm{AX}_{n}$ ) 刻蚀掉相互作用较弱的 $\mathrm{A}$ 层得到(图1) ${ }^{[12 \sim 14]}$. 其中 $\mathrm{M}$ 代表早期过渡金属(如 $\mathrm{Sc} 、 \mathrm{Ti} 、 \mathrm{Zr} 、 \mathrm{Hf} 、 \mathrm{~V}$ 、

$\mathrm{Nb} 、 \mathrm{Ta} 、 \mathrm{Cr} 、 \mathrm{Mo}), \mathrm{A}$ 为元素周期表中的 13 和 14 主族 元素, $X$ 为碳或氮, $n=1 ， 2 ， 3^{[12,15 \sim 17]}$. 2011年, Naguib 等 ${ }^{[17]}$ 使用氢氟酸选择性蚀刻 $\mathrm{Ti}_{3} \mathrm{AlC}_{2}$ 中的 $\mathrm{Al}$, 发现了第 一例MXene化合物 $\mathrm{Ti}_{3} \mathrm{C}_{2} \mathrm{~T}_{x}, \mathrm{~T}_{x}$ 代表刻蚀后引入的-O、 $-\mathrm{F}$ 以及- $\mathrm{OH}$ 等表面官能团. 由于具有优异的物理化学 性质，随后的几年里MXene材料得到了快速发展，已 被广泛应用于储能 ${ }^{[18 ~ 21]}$ 、海水淡化 ${ }^{[22]}$ 、催化 ${ }^{[23]}$ 、电

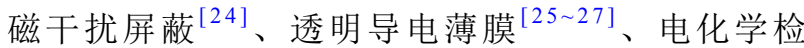
测 ${ }^{[28,29]}$ 等领域. 对于放射性核素以及重金属离子吸附 应用，MXene材料表现出较大的比表面积、丰富的活 性位点、较高的离子交换容量、可控的层间距以及良 好的亲水性等优点. 更重要的是, MXene材料很好地继 承了母体材料MAX相所具有的化学与结构稳定性，以 及优良的导热性和抗辐照性能 ${ }^{[30 \sim 32]}$, 因此除了能够清 除重金属离子等普通环境污染物，对于高释热、强放 射环境下核废物的处置也具有实际的应用价值. 此外, 目前能够成功合成的MXene类型多于20种，根据元素 组成和层结构的不同它们的性质也各异，因此将这类

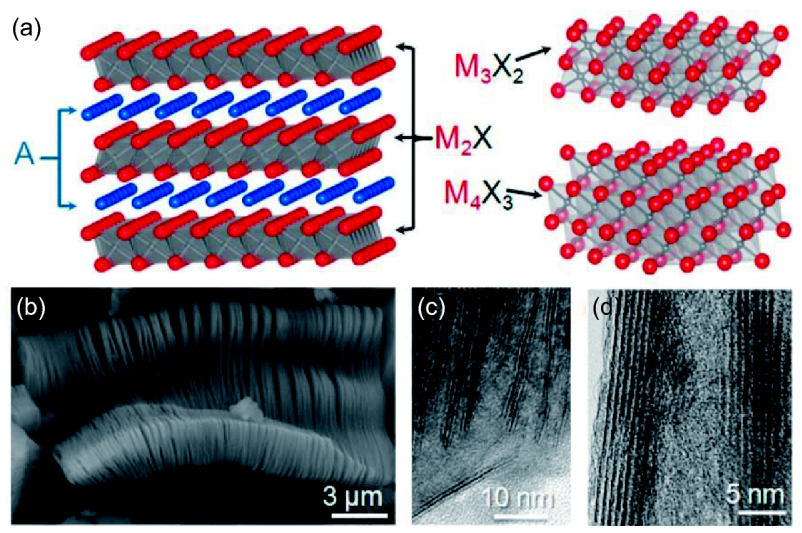

图 1 MXene材料的结构和形貌图 ${ }^{[12 ~ 14]}$. (a) MAX相及 MXene结构示意图; (b) $\mathrm{Ti}_{3} \mathrm{C}_{2} \mathrm{~T}_{x}$ 型MXene片层材料的扫描电 子显微镜 $(\mathrm{SEM})$ 照片; (c, d) $\mathrm{Nb}_{2} \mathrm{CT}_{x}$ 和 $\mathrm{Ti}_{3} \mathrm{C}_{2} \mathrm{~T}_{x}$ 片层材料的透 射电子显微镜(TEM)照片(网络版彩图)

Figure 1 Structure and morphology of MXene [12-14]. (a) Schematics of the MAX crystal structure and MXene layers; (b) SEM image of $\mathrm{Ti}_{3} \mathrm{C}_{2} \mathrm{~T}_{x} ;(\mathrm{c}, \mathrm{d})$ cross-sectional TEM images of $\mathrm{Nb}_{2} \mathrm{CT}_{x}$ sheets and $\mathrm{Ti}_{3} \mathrm{C}_{2} \mathrm{~T}_{x}$ scroll, respectively (color online).

材料用于放射性核素与重金属离子的吸附分离，可选 择性强，便于通过系统研究篮选出性能优异的MXene 吸附剂材料. 基于此，本文主要综述MXene材料的可 控制备与基本性质，以及它们在放射性核素和有毒重 金属离子去除领域的研究进展，并对相关应用前景做 了展望.

\section{MXene的可控制备与基本性质}

\section{1 可控制备}

尽管MAX相中A层的反应活性高于 $\mathrm{M}_{n+1} \mathrm{X}_{n}$ 层, $\mathrm{M}-$ $\mathrm{A}$ 的成键性质仍为金属键，其相互作用强度远大于常 规二维材料(如石墨烯、氮化硼以及过渡金属二硫化 物)层间的弱范德华力, 因此传统的机械剥离或者直接 超声方式已不适用，必须使用强腐蚀性的化学刻蚀剂 (如HF水溶液)去除A层来获得MXene. 根据母体材料 稳定性的不同，完成刻蚀所需的适宜HF浓度以及反应 温度也有着较大差异 ${ }^{[14]}$. 使用含有 $\mathrm{HF}$ 的盐(如 $\mathrm{NH}_{4} \mathrm{HF}_{2}$ ) 或者可以原位形成HF的反应体系(如氟盐和盐酸溶液 混合物)能够一步得到阳离子插层的MXene材料 ${ }^{[18,33]}$, 该刻蚀方法所需的氟离子浓度较低, 因此也更为绿色 环保. Alhabeb 等 ${ }^{[34]}$ 发展了一种 $\mathrm{H}_{2} \mathrm{O}_{2}$ 辅助的 $\mathrm{HF}$ 刻蚀方 法, 以 $\mathrm{Ti}_{3} \mathrm{SiC}_{2}$ 作为前体, 成功制备了具有较强抗氧化 能力的 $\mathrm{Ti}_{3} \mathrm{C}_{2} \mathrm{~T}_{x}$. 而对于结构稳定的高 $n \mathrm{MAX}$ 材料则可 
使用含氟熔盐体系制备MXene. 例如，Urbankowski 等 ${ }^{[35]}$ 在 $550^{\circ} \mathrm{C}$ 的熔盐体系中对 $\mathrm{Ti}_{4} \mathrm{AlN}_{3}$ 刻蚀得到了 $\mathrm{Ti}_{4}$ $\mathrm{N}_{3} \mathrm{~T}_{x}$. 鉴于铝易与碱溶液反应的特性, $\mathrm{Li}^{\text {等 }}{ }^{\left[{ }^{[6]}\right.}$ 采用高浓 度 $\mathrm{NaOH}$ 水热法成功制备了无氟端基的多层 $\mathrm{Ti}_{3} \mathrm{C}_{2} \mathrm{~T}_{x}$, 该材料表面基团全部为 $-\mathrm{O} /-\mathrm{OH}$, 相较于含氟体系制备 的MXene能够提供更多的活性吸附位点. 此外, 对于某 些特定的非MAX相三元金属陶瓷材料亦可使用选择 性刻蚀的方法制备MXene材料. 例如, Zhou等 ${ }^{[37,38]}$ 使用 $\mathrm{HF}$ 选择性刻蚀 $\mathrm{Zr}_{3} \mathrm{Al}_{3} \mathrm{C}_{5}$ 与 $\mathrm{Hf}_{3}[\mathrm{Al}(\mathrm{Si})]_{4} \mathrm{C}_{6}$ 中的 $\mathrm{Al}_{3} \mathrm{C}_{3}$ 和 $\mathrm{Al}(\mathrm{Si})]_{4}$ 层, 分别得到了 $\mathrm{Zr}_{3} \mathrm{C}_{2} \mathrm{~T}_{x}$ 和 $\mathrm{Hf}_{3} \mathrm{C}_{2} \mathrm{~T}_{x}$ 型MXene. 可 以看到, MXene材料通常在强腐蚀、强酸、强碱、高 温或者强氧化等条件下制备得到, 化学与结构稳定性 好, 抗极端环境能力强, 因此在环境污染物的处理应 用方面具有优良的耐候性, 此外还可以根据特定环境 条件选取合适的MXene制备方法.

\section{2 基本性质}

MXene的刻蚀过程伴随着 $\mathrm{M}-\mathrm{A}$ 键的断裂以及新 的悬挂键的形成，常见的MXene过渡金属端基如- $\mathrm{O}$ 、 $-\mathrm{OH}$ 以及 $-\mathrm{F}^{[39]}$, 在盐酸-氟盐体系下制备的MXene材料 表面还含有一定量的 $-\mathrm{Cl}^{[40]}$, 这些端基的存在为金属离 子的吸附提供了大量的活性位点. 同时, MXene具有良 好的亲水性以及负的表面电荷 $\left(\right.$ 如 $\mathrm{Ti}_{3} \mathrm{C}_{2} \mathrm{~T}_{x}$ 的零电荷点 $\left.\mathrm{pH}_{\mathrm{PZC}}<3.0\right)$, 水溶液中的阳离子与之接触时会发生自 发插层现象 ${ }^{[20,41]}$, 这对于金属阳离子的吸附非常有利. 此外，使用无机碱或者有机碱进行插层活化时能够改 变MXene表面功能基团的相对含量 ${ }^{[42]}$. 新鲜的多层 MXene还具有类似于天然黏土矿物的柔韧性以及可溶 胀性, 因此一些中性的有机小分子, 如尿素、二甲基亚 砜(DMSO) 以及 $\mathrm{N}_{2} \mathrm{H}_{4}$ 等也可以对其实现插层 ${ }^{[43]}$. 由于 MXene的层间力以弱的范德华相互作用为主，通过水 合阳离子以及有机小分子的插层活化可以实现MXene 的层间距以及层间相互作用的双重设计调控. 例如, 盐 酸-氟化锂体系制备得到的 $\mathrm{Ti}_{3} \mathrm{C}_{2} \mathrm{~T}_{x}$ 由于水合锂离子的 插层作用其干燥样品的 $c$ 轴晶格常数 $(c-\mathrm{LP})$ 相比于纯 $\mathrm{HF}$ 刻蚀得到的MXene增大了 7 8 $\AA$, 并且其在水化状 态下层间能够容纳更多的水分子, $c$-LP可达 $40 \AA^{[18]}$. 较大的层间距不仅能够促进MXene层间的物质输运, 还可以进一步削弱层间的相互作用，因此更易于剥离 出单片层的MXene. 对于层间相互作用较弱的多层 MXene材料, 通过辅助超声与后续的离心分离可以得
到MXene胶体溶液 ${ }^{[43]}$. 剥离后的单层及薄层MXene材 料具有较大的比表面积, 以 $\mathrm{Ti}_{3} \mathrm{C}_{2} \mathrm{~T}_{x}$ 为例, 其理论比表 面积可达 $484 \mathrm{~m}^{2} / \mathrm{g}^{[20]}$, 实验上测量得到的 $\mathrm{Ti}_{3} \mathrm{C}_{2} \mathrm{~T}_{x}$ 薄层 以及 $\mathrm{Ti}_{3} \mathrm{C}_{2} \mathrm{~T}_{x}$ 气凝胶的比表面积分别为 $98^{[20]}$ 和 $176 \mathrm{~m}^{2} / \mathrm{g}^{[44]}$. 较大的比表面积使得MXene材料克服了 层间距带来的限制, 其表面活性位点得到充分利用, 从而改善材料的吸附性能. 例如, Shahzad 等 ${ }^{[45]}$ 对多层 $\mathrm{Ti}_{3} \mathrm{C}_{2} \mathrm{~T}_{x}$ MXene进行剥层, 得到的剥层后的薄层 $\mathrm{Ti}_{3} \mathrm{C}_{2} \mathrm{~T}_{x}$ MXene (DL- $\mathrm{Ti}_{3} \mathrm{C}_{2} \mathrm{~T}_{x} \mathrm{MXene}$ ) 展现了对 $\mathrm{Cu}$ (II) 更好的吸 附性能.

\section{MXene材料的吸附研究技术}

\section{1 理论计算}

通过理论计算, 如密度泛函理论 $(\mathrm{DFT})^{[46 \sim 48]}$ 以及 第一性原理 ${ }^{[49,50]}$, 可以深入了解金属离子与MXene材 料之间的吸附机理. DFT是在原子水平上解释或补充 实验结果的最有效的理论工具之一 ${ }^{[51]}$, 已经被用于研 究MXene材料与放射性核素及有毒重金属的吸附机 理, 可以计算最稳定的吸附构象、体系总能量、电荷 密度以及解释可能的吸附机制 ${ }^{[46-48]}$. 例如, Zhang 等 ${ }^{[48]}$ 通过DFT探究了MXene材料 $\left(\mathrm{V}_{2} \mathrm{CT}_{x}\right)$ 和放射性核素 $(\mathrm{U}$ (VI))之间的相互作用机理, 计算了体系的总能量信 息、电荷密度, 得到了MXene材料对放射性核素的吸 附性能、吸附构型、其他离子干扰等结果. 这些结果 可以帮助研究人员设计相应的MXene材料, 控制和优 化对放射性核素的吸附性能.

\section{2 批次实验}

批次实验技术是目前应用最广泛的技术之一，具 有方便和高效的特点, 可以提供有关吸附过程中的平 衡时间、最大吸附容量、最适宜 $\mathrm{pH}$ 以及吸附选择性 等有价值的信息 ${ }^{[52,53]}$.

\subsection{1 动力学实验}

动力学实验可以提供吸附反应时间和平衡时间等 信息. 吸附动力学实验结果显示, MXene材料对 U(VI) 的吸附平衡时间为4.5 6 $\mathrm{h}^{[46 \sim 48]}$, 对 $\mathrm{Pb}$ (II)、 $\mathrm{Cu}(\mathrm{II})$ 、 $\mathrm{Ba}(\mathrm{II}) 、 \mathrm{Cr}(\mathrm{VI}) 、 \mathrm{Hg}(\mathrm{II})$ 的平衡时间分别为 200 $\min ^{[54]} 、 5 \mathrm{~h}^{[45]} 、 120 \mathrm{~min}^{[55]} 、 120 \min ^{[56]}$ 和 $300 \min ^{[57]}$. 这展示了MXene材料相对于其他材料较快的吸附平衡 
时间.

动力学实验还可以预测潜在的速率控制步骤，包 括质量传递和化学反应过程. 例如, Fard等 ${ }^{[5]}{ }^{-}$研究发 现, MXene材料对 $\mathrm{Ba}(\mathrm{II})$ 的吸附速率先快后慢, 这是因 为其吸附过程包括两个阶段，即钡离子与MXene表面 之间的表面络合(或离子交换)和静电吸引. 吸附过程 刚开始时，MXene的外表面附有大量的空结合位点， $\mathrm{Ba}$ (II)以一种随机的方式占据了许多MXene的活性位 点, 形成化学键. 当MXene的外表面活性位点被钡离子 耗尽和饱和后, $\mathrm{Ba}(\mathrm{II})$ 的吸收速率降低, 达到平衡.

通常使用准一阶模型(PFO)和准二阶模型(PSO)对 核素及其重金属在MXene材料上的吸附动力学进行准 确研究, 结果大部分符合准二阶模型(PSO) $)^{[45,52 \sim 55,57]}$, 这表明吸附过程主要是化学吸附，而不是物理吸附. 这两种模型(PFO和PSO)缺点在于, 都不能用来判断相 关吸附过程中的主导速率限制是外部扩散还是内部扩 散 ${ }^{[58]}$.

\subsection{2 热力学实验}

通过热力学实验得到吸附等温线，可以获得吸附 剂的最大吸附容量、吸附机理以及吸附剂的表面性质 等信息 ${ }^{[5]}$. 使用Langmuir、Freundlich、Redlich-Peterson、Dubinin-Radushkevich (D-R)和Temkin等多种吸 附等温线模型对基于MXene材料的吸附等温线进行模 拟研究.

$\mathrm{Gu}$ 等 ${ }^{[54]}$ 的研究结果显示, $\mathrm{Pb}(\mathrm{II})$ 在 $\mathrm{Ti}_{3} \mathrm{AlC}_{2}$ 纳米纤 维( $\left(\mathrm{e}-\mathrm{TACFs}\right.$ ) 和 $\mathrm{Ti}_{3} \mathrm{AlC}_{2}$ 纳米片 (e-TACSs)上的吸附等温 线模型, 都符合Langmuir模型, 表明其吸附行为都属于 单分子层吸附. Shahzad等 ${ }^{\left[{ }^{[7]}\right.}$ 研究结果表明, $\mathrm{Hg}(\mathrm{II})$ 在磁 性二维层状 $\mathrm{Ti}_{3} \mathrm{C}_{2} \mathrm{~T}_{x}$ MXene上的吸附等温线模型符合 Redlich-Peterson模型. Fard等 ${ }^{[55]}$ 和Shahzad 等 ${ }^{[45]}$ 的研究

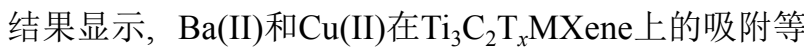
温线模型符合Freundlich模型，表明吸附过程发生在 MXene在多层面上的异质表面. Wang 等 ${ }^{[53]}$ 研究结果显

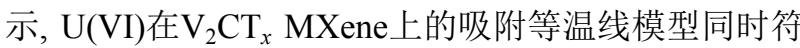
合Freundlich模型和D-R模型. 符合Freundlich模型是因 为 $\mathrm{V}_{2} \mathrm{CT}_{x}$ MXene不仅有多层结构，还有 $-\mathrm{OH} 、-\mathrm{O}$ 和 $-F$ 的表面官能团, 形成了异相吸附位点. 除此之外, 从 D-R模型拟合得出的平均自由能 $11.97 \mathrm{~kJ} / \mathrm{mol}$ 也表明, $\mathrm{U}(\mathrm{VI})$ 和 $\mathrm{V}_{2} \mathrm{CT}_{x}$ 之间的相互作用遵循离子交换机制，这 是一种典型的化学吸附过程 ${ }^{[59,60]}$.
除吸附容量和吸附机理外，还可以从温度相关的 吸附等温线中获得吉布斯自由能变化 $(\Delta G)$ 、焓变 $(\Delta H)$ 和熵变 $(\Delta S)$ 等热力学参数 ${ }^{[5]}$. 这些参数可以进一 步了解金属离子与MXene基材料之间的相互作用行 为 ${ }^{[42,45,54,57]}$. 例如, $\mathrm{Gu}$ 等 $^{[54]}$ 的热力学参数计算结果表 明, e-TACFs 和e-TACSs 对 $\mathrm{Pb}(\mathrm{II})$ 的吸附过程的焓变 $(\Delta H)$ 分别为 15.26 和 $8.84 \mathrm{~kJ} / \mathrm{mol}$, 证明吸附过程为吸热 反应； $298 \mathrm{~K}$ 下的吉布斯自由能变化 $(\Delta G)$ 分别为 -6.94 和 $-5.22 \mathrm{~kJ} / \mathrm{mol}$, 证明了吸附过程都是自发进行的, 而 且 $\mathrm{Pb}(\mathrm{II})$ 在e-TACFs 上的吸附行为比在e-TACSs 上更加 自发.

\section{3 光谱技术}

$\mathrm{X}$ 射线吸收精细结构(XAFS)光谱包括X射线吸收 近缘结构(XANES) 光谱和扩展X射线吸收精细结构 (EXAFS)谱. 通过XAFS光谱测量与分析拟合，可得到 目标元素的价态、目标原子的配位数以及配位层间 距，有助于阐明主要吸附种态和相互作用机理等微观 信息 ${ }^{[22,53]}$. 目前, XAFS技术在MXene吸附过程的微结 构表征中得到了较好的应用 ${ }^{[5]}$. 例如, Wang 等 $^{[53]}$ 为了 确认吸附机理, 在U的初始浓度为 100 和 $200 \mathrm{mg} / \mathrm{L}, \mathrm{pH}$
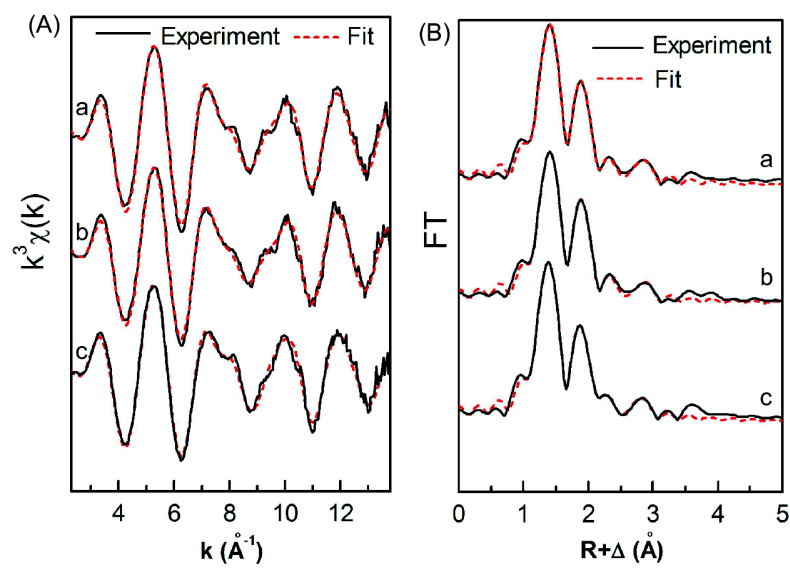

图 2 EXAFS数据拟合图 ${ }^{[33]}$. (A) 在不同溶液 $\mathrm{pH}$ 和初始 $\mathrm{U}(\mathrm{VI})$ 浓度下, 吸附在 $\mathrm{V}_{2} \mathrm{CT}_{x}$ 上的原始 $\mathrm{U} \mathrm{L}_{\mathrm{III}}$-edge $\mathrm{k}^{3}$-weighted 的EXAFS光谱最佳理论拟合图. (a) $\mathrm{pH} 4.5,[\mathrm{U}]_{\text {initial }}=$ $100 \mathrm{mg} / \mathrm{L}$; (b) $\mathrm{pH} 5.0,[\mathrm{U}]_{\text {initial }}=100 \mathrm{mg} / \mathrm{L}$; (c) $\mathrm{pH} 4.2$, $[\mathrm{U}]_{\text {initial }}=200 \mathrm{mg} / \mathrm{L}$. (B) 对应的非相位校正傅里叶变换图谱 (网络版彩图)

Figure 2 Data fitting diagram of EXAFS [53]. (A) Raw $\mathrm{U} \mathrm{L}_{\mathrm{III}}$-edge $\mathrm{k}^{3}$-weighted EXAFS spectra and the best theoretical fits of $\mathrm{U}$ sorbed to $\mathrm{V}_{2} \mathrm{CT}_{x}$ under different solution $\mathrm{pH}$ and initial $\mathrm{U}(\mathrm{VI})$ concentration. (a) $\mathrm{pH} 4.5,[\mathrm{U}]_{\text {initial }}=100 \mathrm{mg} / \mathrm{L} ;$ (b) $\mathrm{pH} 5.0,[\mathrm{U}]_{\text {initial }}=100 \mathrm{mg} / \mathrm{L}$; (c) $\mathrm{pH} 4.2$, $[\mathrm{U}]_{\text {initial }}=200 \mathrm{mg} / \mathrm{L}$. (B) Corresponding nonphase shift corrected Fourier transforms (color online). 
4.2 5 时, 进行了EXAFS测量(图2). 拟合结果表明，铀 酰离子与 $\mathrm{V}_{2} \mathrm{CT}_{x}$ 片层材料钒活性位点上的两个羟基结 合形成了稳定的二齿配合物, 从而实现了对 $\mathrm{U}(\mathrm{VI})$ 的快 速高效吸附. 铀的初始浓度以及反应 $\mathrm{pH}$ 在实验条件中 对反应机理没有显著影响.

$X$ 射线光电子能谱(XPS)分析可以为所有材料表 面和近表面的元素(除了氢外)提供元素氧化态、元素 种类和成键关系等信息 ${ }^{[61]}$. 到目前为止，许多研究已 经使用XPS技术来表征有毒/放射性金属离子的基于 MXene材料的吸附特性 ${ }^{[22,45,54 ~ 57,62]}$. 例如, Peng 等 ${ }^{[42]}{ }$ 研 究了其吸附材料碱化MXene在 $\mathrm{Pb}(\mathrm{II})$ 吸附前后的一些 元素的XPS变化. 纯的 $\mathrm{Pb}\left(\mathrm{NO}_{3}\right)_{2}$ 中 $\mathrm{Pb}(\mathrm{II})$ 的 $\mathrm{Pb} 4 \mathrm{f}_{5 / 2}$ 峰位 在 $144.5 \mathrm{eV}, \mathrm{Pb}_{7} \mathrm{f}_{7 / 2}$ 的峰位在 $139.6 \mathrm{eV}$. 吸附后的碱化 MXene中 $\mathrm{Pb} 4 \mathrm{f}$ 的峰位减小了 $0.8 \mathrm{eV}$ ，这说明 $\mathrm{Pb}(\mathrm{II})$ 离子 和碱化MXene之间形成了强亲和力.

此外, 许多研究人员基于傅里叶变换红外光谱 (FT-IR)对官能团和高度极性键的敏感性进行了研究. 他们利用FT-IR技术将宏观吸附数据与基于MXene材 料的金属离子相互作用结合起来，进行分子规模的研 究 ${ }^{[52,53]}$. Wang 等 ${ }^{[52]}$ 测量了吸附 $\mathrm{U}(\mathrm{VI})$ 前后 $\mathrm{Ti}_{3} \mathrm{C}_{2} \mathrm{~T}_{x}$ MXene的FT-IR光谱, 结果显示, 吸附后的 $\mathrm{Ti}_{3} \mathrm{C}_{2} \mathrm{~T}_{x}$ MXene在 $912 \mathrm{~cm}^{-1}$ 位置出现了铀酰离子的 $\mathrm{U}=\mathrm{O}$ 吸收带, 证明了铀酰离子成功吸附在 $\mathrm{Ti}_{3} \mathrm{C}_{2} \mathrm{~T}_{x}$ 上.

\section{MXene材料的吸附行为与作用机理}

\section{1 放射性元素离子的去除}

铀是一种放射性元素, 具有较强的化学毒性, 进入 人体会导致癌症风险增加以及造成肝脏的损伤 ${ }^{[56]}$. 自 然界中的铀主要以+6价的铀酰离子 $\left(\mathrm{UO}_{2}{ }^{2+}\right)$ 形式存在, 其来源主要是铀矿开采以及核工业生产 ${ }^{[63]}$. 鉴于 $U(V I)$ 具有较强的迁移性, 环境中U(VI)放射性污染的治理非 常重要和关键. 本文中, 我们将MXene材料对 U(VI)的 吸附作用和机理进行系统总结.

由于吸附剂MXene中存在不同的吸附位点(如 $\mathrm{OH} 、 \mathrm{O}$ 和F), MXene对 $\mathrm{U}(\mathrm{VI})$ 的吸附过程遵循非均质吸 附模型. 相较于传统的无机纳米材料, MXene材料是一 种高效的放射性核素吸附剂, 吸附平衡时间短, 吸附容 量大. 例如, 多层 $\mathrm{V}_{2} \mathrm{CT}_{x}$ MXene, 在 $\mathrm{pH}$ 3 5的范围内, 对 $\mathrm{U}(\mathrm{VI})$ 的最大吸附容量为 $174 \mathrm{mg} / \mathrm{g}$, 吸附平衡时间为 $6 \mathrm{~h}^{[53]}$. 水化插层 $\mathrm{Ti}_{3} \mathrm{C}_{2} \mathrm{~T}_{x}$ MXene, 在 $\mathrm{pH}$ 为 5 时对 $\mathrm{U}(\mathrm{VI})$
的最大吸附容量为 $214 \mathrm{mg} / \mathrm{g}$, 吸附平衡时间为 $4.5 \mathrm{~h}^{[52]}$. 为了充分利用MXene的层间空间, Wang 等 ${ }^{[52]}$ 通过水化 插层的方法扩大了 $\mathrm{Ti}_{3} \mathrm{C}_{2} \mathrm{~T}_{x}$ MXene的层间距, 增加了其 对U(VI)的吸附, 随后通过低温快速炦烧的方法将吸附 后的 $\mathrm{U}(\mathrm{VI})$ 封存在 $\mathrm{Ti}_{3} \mathrm{C}_{2} \mathrm{~T}_{x}$ 的层间, 实现了对放射性 $\mathrm{U}(\mathrm{VI})$ 的固定化(图3). 其脱附实验结果显示, 经过炦烧 后的 $\mathrm{Ti}_{3} \mathrm{C}_{2} \mathrm{~T}_{x}$ 中的 $\mathrm{U}(\mathrm{VI})$ 在水溶液中的溶出率小于 $1 \%$, 在 $0.5 \mathrm{M}$ 硝酸中溶出率小于 $6 \%$, 成功实现了放射性核 素的固定封装.

$\mathrm{V}_{2} \mathrm{CT}_{x}$ MXene材料对铀离子的吸附去除机理主要 是离子交换和配位作用. DFT计算和EXAFS结果都表 ${ }^{\text {明 }}{ }^{[53]}, \mathrm{U}(\mathrm{VI})$ 与纳米片 $\mathrm{V}$ 活性位点上的羟基结合, 形成 了双齿内层配合物. $\mathrm{V}_{2} \mathrm{CT}_{x}$ 与 $\mathrm{U}(\mathrm{VI})$ 结合后羟基的去质 子化表明吸附过程为离子交换机制. Zhang 等 ${ }^{[48]}$ 通过 电子态密度、差分电荷密度等手段分析, 揭示出化学 键作用和氢键形成是 $\mathrm{V}_{2} \mathrm{C}(\mathrm{OH})_{2}$ 层状材料对铀离子吸 附稳定的主要作用. 同时研究了 $\mathrm{V}_{2} \mathrm{CT}_{x}$ 层状材料的其 他功能化表面基团对铀离子吸附能力的影响. 铀离子 主要通过如下两种形式的相互作用稳定吸附在 $\mathrm{V}_{2} \mathrm{C}$ 层 状材料上：铀原子与表面基团的化学键作用和铀离子 基团的轴向氧与表面基团的弱相互作用. 并预测出 $-\mathrm{F}$ 功能化基团对铀离子的吸附起到一定抑制作用.

$\mathrm{Ti}_{3} \mathrm{C}_{2} \mathrm{~T}_{x}$ MXene材料对铀离子的吸附作用机理与 $\mathrm{V}_{2} \mathrm{CT}_{x}$ MXene相似. Zhang等 ${ }^{[50]}$ 计算结果表明, 铀酰离 子与去质子化后的羟基 $\mathrm{Ti}_{3} \mathrm{C}_{2} \mathrm{~T}_{x}$ 表面上的两个氧原子 以共价键的形式相结合时，整个结构的能量最低，即

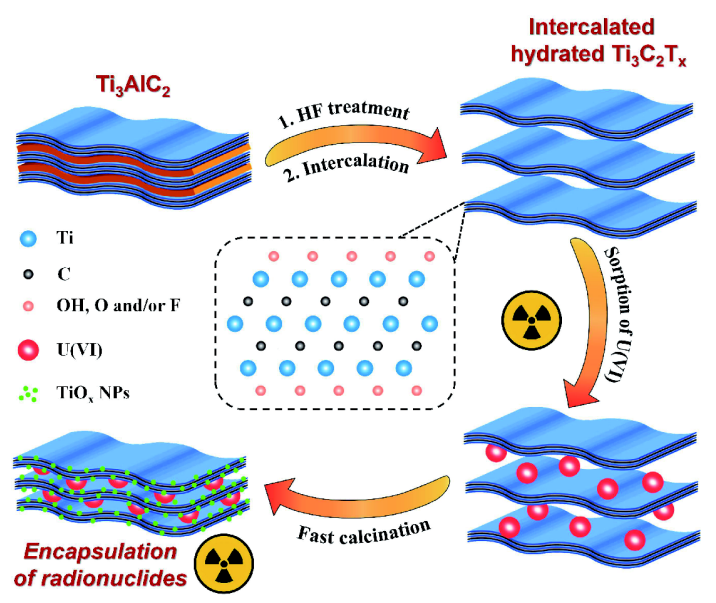

图 3 通过水化插层的方法将 $\mathrm{U}(\mathrm{VI})$ 吸附并封存在 $\mathrm{Ti}_{3} \mathrm{C}_{2} \mathrm{~T}_{x}$ MXene的层间示意图 ${ }^{[52]}$ (网络版彩图)

Figure 3 Hydrated intercalation synthesis strategy of $\mathrm{Ti}_{3} \mathrm{C}_{2} \mathrm{~T}_{x}$ MXene for efficient $\mathrm{U}(\mathrm{VI})$ uptake and imprisonment [52] (color online). 
$\left[\mathrm{UO}_{2}\left(\mathrm{H}_{2} \mathrm{O}\right)_{5}\right]^{2+}$ 首先脱去两个内配位的水分子，然后与 去质子化的 $\mathrm{Ti}_{3} \mathrm{C}_{2} \mathrm{~T}_{x}$ 表面端氧原子形成稳定的二齿内 层配合物(图4). 此外还考虑了其他阴离子配体(如 $\mathrm{OH}-、 \mathrm{Cl}-$ 和 $\mathrm{NO}_{3}{ }^{-}$) 对吸附过程的影响, 结果表明, 这 些配体参与配位的情况下, 铀酰离子与羟基化 $\mathrm{Ti}_{3} \mathrm{C}_{2} \mathrm{~T}_{x}$ 的结合仍然保持着二齿配位的稳定构型.

在已有的报道中, 研究者总结了纳米零价铁 ${ }^{[64]}$ 、 碳基纳米材料 ${ }^{[65]}$ 、金属氧化物材料 ${ }^{[66]}$ 、四氧化三铁 基纳米材料 ${ }^{[67]}$ 以及金属有机骨架化合物(MOFs) ${ }^{[5]}$ 等 材料对U(VI)的吸附行为和吸附机理. 本文选取几种常 见的代表性吸附剂材料, 将其与MXene对U(VI)的吸附 行为进行简要对比(表1). 可以看出, 不同吸附材料对 $\mathrm{U}(\mathrm{VI})$ 的吸附机理主要有离子交换、配位作用、氧化 还原和共沉淀等, MXene材料对U(VI)的吸附机理主要 是离子交换和配位作用的协同机制. 同时, MXene材料 对U(VI)的吸附容量显著高于常见的金属氧化物纳米 颗粒以及高岭土、蒙脱土等天然层状材料. 以石墨烯 为代表的碳基材料表面具有丰富的官能团, 可修饰性 强, 因此相比MXene展现了更大的U(VI)吸附容量.

\section{2 有毒重金属离子的去除}

MXene材料有着较大的比表面积和丰富的表面官
能团, 提供了较多的重金属离子吸附位点, 已经在重金 属吸附领域有了广泛的应用(表2). Guo等 ${ }^{[28]}$ 使用DFT 解释了重金属离子在MXene上的吸附动力学和MXene 夹层对吸附的影响, 计算结果显示, 碱化后的二维层状 MXene材料 $\mathrm{Ti}_{3} \mathrm{C}_{2}(\mathrm{OH})_{2}$ 可以实现重金属离子的高效吸 附. 重金属原子周围的 $\mathrm{Ti}_{3} \mathrm{C}_{2}(\mathrm{OH})_{2}$ 上的氢原子和重金 属原子上的 $\mathrm{O}$ 原子组成了氢势阱, 提供了足够的相反 自旋电子对以捕获重金属原子. 此外, $\mathrm{F}$ 和其他氢离子 的残留会削弱碱化MXene $\left(\mathrm{Ti}_{3} \mathrm{C}_{2}(\mathrm{OH})_{2}\right)$ 的离子吸附效 率, $\mathrm{Li}^{+} 、 \mathrm{Na}^{+}$和 $\mathrm{K}^{+}$的插层会使得碱化 MXene $\left(\mathrm{Ti}_{3} \mathrm{C}_{2}{ }^{-}\right.$ $\left.(\mathrm{OH})_{2}\right)$ 的离子吸附效率增强. 更重要的是, 钛原子的羟 基垂直位置显示出比其他位置更强的金属离子去除 趋势.

\subsection{1 $\mathrm{Pb}(\mathrm{II})$ 的去除}

$\mathrm{Pb}(\mathrm{II})$ 是一种不可生物降解的有毒重金属. 2014 年, Peng 等 ${ }^{[42]}$ 采用 $\mathrm{NaOH}$ 碱化插层的方法制备了碱化 MXene材料用于水体中 $\mathrm{Pb}(\mathrm{II})$ 的吸附. 这种碱化MXene 材料具有很强的吸附能力、快速的动力学、极微量的 $\mathrm{Pb}(\mathrm{II})$ 流出物和可逆的吸附特性. $1 \mathrm{~kg}$ 碱化MXene材料 可以处理 $4500 \mathrm{~kg}$ 含铅水, 处理后水体 $\mathrm{Pb}(\mathrm{II})$ 的含量低 于世界卫生组织标准 $(10 \mu \mathrm{g} / \mathrm{L})$, 在较高浓度阳离子

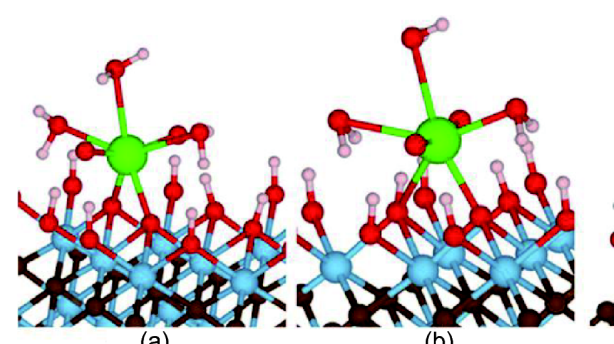

(a)

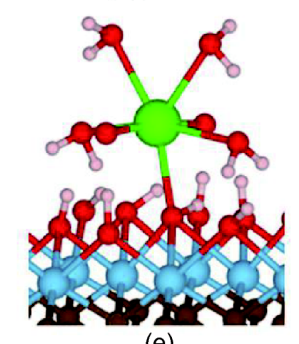

(e)

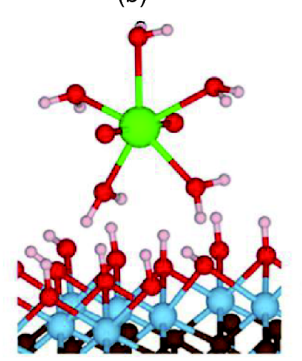

(f)

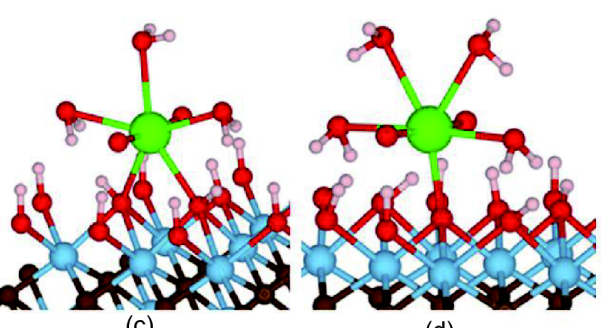

(c)

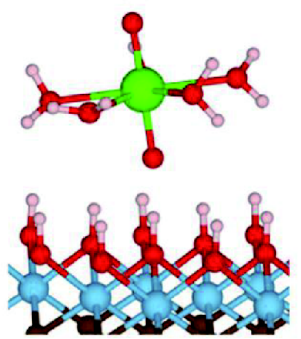

(g)

(d)

图 $4 \mathrm{Ti}_{3} \mathrm{C}_{2} \mathrm{~T}_{x}$ 与U(VI)相互作用的模型示意图 ${ }^{[50]}$. (a) 去质子化双齿配位; (b) 半质子化双齿配位; (c) 质子化双齿配位; (d) 去质 子化单齿配位; (e) 质子化单齿配位; (f) 赤道面外层配位; (g) 端氧外层配位(网络版彩图)

Figure 4 Snapshots of uranyl inner- and outer-sphere adsorption configurations on $\mathrm{Ti}_{3} \mathrm{C}_{2}(\mathrm{OH})_{2}$ nanosheet $[50]$. (a) $\mathrm{TiO}_{2}-\mathrm{UO} \mathrm{O}_{2}\left(\mathrm{H}_{2} \mathrm{O}\right)_{3}$, (b) TiO(OH)$\mathrm{UO}_{2}\left(\mathrm{H}_{2} \mathrm{O}\right)_{3}$ and (c) Ti(OH) $-\mathrm{UO}_{2}\left(\mathrm{H}_{2} \mathrm{O}\right)_{3}$ are uranyl bidentate adsorption configurations; (d) TiO- $\mathrm{UO}_{2}\left(\mathrm{H}_{2} \mathrm{O}\right)_{4}$ and $(\mathrm{e})$ TiOH-UO $\mathrm{U}_{2}\left(\mathrm{H}_{2} \mathrm{O}\right)_{4}$ are uranyl monodentate adsorption configurations; (f) $\left[\mathrm{TiOH} \cdots \mathrm{UO}_{2}\left(\mathrm{H}_{2} \mathrm{O}\right)_{5}\right]_{p}$ and $(\mathrm{g})\left[\mathrm{TiOH} \cdots \mathrm{UO}_{2}\left(\mathrm{H}_{2} \mathrm{O}\right)_{5}\right]_{v}$ are uranyl outer-sphere adsorption configurations (color online). 
表 1 MXene以及其他纳米材料对U(VI)的去除效果

Table 1 Removal of U(VI) by MXene and other nanomaterials

\begin{tabular}{|c|c|c|c|c|c|c|}
\hline 核素 & 吸附材料 & $Q_{\max }(\mathrm{mg} / \mathrm{g})$ & $m / V(\mathrm{~g} / \mathrm{L})$ & $\mathrm{pH}$ & 作用机理 & 参考文献 \\
\hline \multirow{11}{*}{$\mathrm{U}(\mathrm{VI})$} & 水合 $\mathrm{Ti}_{3} \mathrm{C}_{2} \mathrm{~T}_{x}$ & 214 & 0.4 & 5.0 & $\begin{array}{c}\text { 离子交换、 } \\
\text { 配位作用 }\end{array}$ & {$[50,52]$} \\
\hline & $\mathrm{V}_{2} \mathrm{CT}_{x}$ & 174 & 0.4 & 5.0 & $\begin{array}{c}\text { 离子交换、 } \\
\text { 配位作用 }\end{array}$ & {$[48,53]$} \\
\hline & 纳米零价铁 & 122.6 & - & 5.5 & 氧化还原共沉淀 & [64] \\
\hline & $\mathrm{Fe}_{3} \mathrm{O}_{4} @ \mathrm{GO}$ & 69.5 & 0.3 & 5.5 & 配位作用 & {$[68]$} \\
\hline & GO & 299 & 0.4 & 4.0 & 配位作用 & [69] \\
\hline & Nano- $-\mathrm{Fe}_{3} \mathrm{O}_{4}$ & 80.9 & 0.2 & 5.0 & - & {$[70]$} \\
\hline & UiO-66 & 109.9 & 0.4 & 5.5 & 离子交换 & {$[71]$} \\
\hline & UiO-66- $\mathrm{NH}_{2}$ & 114.9 & 0.4 & 5.5 & 离子交换 & [71] \\
\hline & MOF-3 & 109 & 1.0 & 2.5 & 离子交换 & [7] \\
\hline & 高岭土 & 20 & 5.0 & 5.2 & 配位作用 & {$[72]$} \\
\hline & 蒙脱石 & 9.6 & 2.0 & 6.8 & 配位作用 & [10] \\
\hline
\end{tabular}

表 2 MXene及其复合物在核素和有毒重金属吸附中的应用

Table 2 The application of MXene and its complex in the adsorption of toxic and radioactive metals

\begin{tabular}{|c|c|c|c|c|c|c|}
\hline 目标离子 & 吸附材料 & 吸附容量(mg/g) & 平衡时间 & $\mathrm{pH}$ & 机理 & 参考文献 \\
\hline \multirow{2}{*}{$\mathrm{U}(\mathrm{VI})$} & 水合 $\mathrm{Ti}_{3} \mathrm{C}_{2} \mathrm{~T}_{x}$ & 214 & $6 \mathrm{~h}$ & 5.0 & $\begin{array}{l}\text { 离子交换 } \\
\text { 配位作用 }\end{array}$ & {$[50,52]$} \\
\hline & $\mathrm{V}_{2} \mathrm{CT}_{x}$ & 174 & $4.5 \mathrm{~h}$ & 5.0 & $\begin{array}{l}\text { 离子交换 } \\
\text { 配位作用 }\end{array}$ & {$[48,53]$} \\
\hline \multirow{3}{*}{$\mathrm{Pb}(\mathrm{II})$} & $\begin{array}{c}\text { 碱化MXene }\left(\mathrm{Ti}_{3} \mathrm{C}_{2}-\right. \\
\left.(\mathrm{OH} / \mathrm{ONa})_{x} \mathrm{~F}_{2-x}\right)\end{array}$ & 140 & $2 \min$ & 6.5 & 离子交换 & [42] \\
\hline & $\begin{array}{c}\mathrm{Ti}_{3} \mathrm{AlC}_{2} \text { 纳米纤维 } \\
(\mathrm{e}-\mathrm{TACFs})\end{array}$ & 285.9 & $20 \mathrm{~min}$ & 5.0 & 离子交换 & [54] \\
\hline & $\begin{array}{c}\mathrm{Ti}_{3} \mathrm{AlC}_{2} \text { 纳米片 } \\
\text { (e-TACSs) }\end{array}$ & 218.3 & $200 \mathrm{~min}$ & 5.0 & 离子交换 & [54] \\
\hline \multirow[t]{2}{*}{$\mathrm{Cu}(\mathrm{II})$} & 薄层 $\mathrm{Ti}_{3} \mathrm{C}_{2} \mathrm{~T}_{x} \mathrm{MXene}$ & 78.5 & $5 \mathrm{~h}$ & 5.0 & $\begin{array}{l}\text { 离子交换 } \\
\text { 氧化还原 }\end{array}$ & [45] \\
\hline & 水合插层MXene & 45 & $120 \mathrm{~min}$ & 5.0 & - & {$[52]$} \\
\hline \multirow{2}{*}{$\mathrm{Ba}(\mathrm{II})$} & $\mathrm{Ti}_{3} \mathrm{C}_{2} \mathrm{~T}_{x}$ MXene & 9.3 & $60 \mathrm{~min}$ & 6.0 & 离子交换 & [55] \\
\hline & 碱化 $\mathrm{Ti}_{3} \mathrm{C}_{2} \mathrm{~T}_{x} \mathrm{MXene}$ & 46.5 & $24 \mathrm{~h}$ & 7.0 & 离子交换 & [73] \\
\hline \multirow{2}{*}{$\mathrm{Cr}(\mathrm{VI})$} & $\mathrm{Ti}_{3} \mathrm{C}_{2} \mathrm{~T}_{x} \mathrm{MXene}$ & 250 & $2 \mathrm{~h}$ & 5.0 & $\begin{array}{l}\text { 静电吸附 } \\
\text { 氧化还原 }\end{array}$ & [62] \\
\hline & $\begin{array}{c}\mathrm{TiO}_{2}-\mathrm{C}(\mathrm{u}-\mathrm{RTC}) \text { 复 } \\
\text { 合材料 }\end{array}$ & 225 & $120 \mathrm{~min}$ & 6.0 & 配位作用 & {$[56]$} \\
\hline $\mathrm{Cd}(\mathrm{II})$ & 水合插层MXene & 35 & $60 \mathrm{~min}$ & 5.0 & - & {$[52]$} \\
\hline $\mathrm{Hg}(\mathrm{II})$ & 磁性 $\mathrm{Ti}_{3} \mathrm{C}_{2} \mathrm{~T}_{x} \mathrm{MXene}$ & 1128.4 & $300 \mathrm{~min}$ & 6.0 & 静电吸附 & [57] \\
\hline
\end{tabular}

$(\mathrm{Ca}(\mathrm{II}) / \mathrm{Mg}(\mathrm{II}))$ 存在时依然对 $\mathrm{Pb}(\mathrm{II})$ 有很好的选择性. 动 力学实验表明, 吸附平衡时间为 $120 \mathrm{~s}$. 实验和计算研
究表明, 吸附行为与活化的 $\mathrm{Ti}$ 位点中的羟基有关, MXene材料特有的分层结构以及活化后丰富的钛羟基 
为环境修复中的 $\mathrm{Pb}$ (II)净化提供了潜在的应用. 2016 年, Guo等 ${ }^{[46]}$ 通过第一性原理研究了 $\mathrm{Pb}(\mathrm{II})$ 在MXene材 料表面的吸附行为. 对MXene材料在吸附体系中的计 算结果表明，由于相似的层能量，不同 $\mathrm{OH}$ 基团位置位 点的3种MXene结构可以共存于溶液中, 其中两个羟基 处在过渡金属 $\mathrm{M}$ 位点上的构型是最为稳定的一种构型 (除了 $\mathrm{Zr}_{2} \mathrm{C} 、 \mathrm{Nb}_{2} \mathrm{~N} 、 \mathrm{Hf}_{2} \mathrm{C} 、 \mathrm{Ta}_{2} \mathrm{C}$ 和 $\mathrm{Ta}_{2} \mathrm{~N}$ ). 通过对 3 种模 型的计算分析证明, 除了 $\mathrm{Sc}_{2} \mathrm{C}(\mathrm{OH})_{2}$ 和 $\mathrm{Zr}_{2} \mathrm{C}(\mathrm{OH})_{2}$ 对 $\mathrm{Pb}$ (II)没有吸附能力外, 其余所有 $\mathrm{M}_{2} \mathrm{X}(\mathrm{OH})_{2}$ 结构的 MXene材料对 $\mathrm{Pb}$ (II) 都有吸附去除能力, 其中 $\mathrm{Ti}_{2} \mathrm{C}$ $(\mathrm{OH})_{2}$ 对于重金属离子的去除效果最好. 2017年, $\mathrm{Gu}$ 等 ${ }^{[54]}$ 使用不含氟的强碱 $\mathrm{NaOH}$ 刻蚀MAX材料 $\mathrm{Ti}_{3} \mathrm{AlC}_{2}$, 得到 $\mathrm{Ti}_{3} \mathrm{AlC}_{2}$ 纳米纤维 $\left(\mathrm{e}-\mathrm{TACFs}\right.$ ) 和 $\mathrm{Ti}_{3} \mathrm{AlC}_{2}$ 纳米片 $(\mathrm{e}-$ TACSs) (图5). 实验结果和理论计算证明了 e-TACFs和 e-TACSs这两种材料对 $\mathrm{Pb}(\mathrm{II})$ 具有很好的吸附性能, 由 温度相关的等温线计算出的热力学参数表明, 两种样 品对 $\mathrm{Pb}(\mathrm{II})$ 的吸附都是自发的和吸热的. e-TACFs和eTACSs 具有高反应活性和强络合能力, 在 $\mathrm{pH} 5.0$ 的条 件下对 $\mathrm{Pb}$ (II)的吸附容量分别为 285.9 和 $218.3 \mathrm{mg} / \mathrm{g}$. 这 种不含氟的合成路径以及高吸附容量使得 $\mathrm{Ti}_{3} \mathrm{AlC}_{2}$ 纳 米纤维( $(\mathrm{e}-\mathrm{TACFs})$ 和 $\mathrm{Ti}_{3} \mathrm{AlC}_{2}$ 纳米片(e-TACSs) 有望成为 理想的 $\mathrm{Pb}(\mathrm{II})$ 吸附材料.

\subsection{2 $\mathrm{Cu}(\mathrm{II})$ 的去除}

$\mathrm{Cu}(\mathrm{II})$ 与 $\mathrm{Pb}(\mathrm{II})$ 类似，同样具有不可生物降解性和 生物毒性. Shahzad等 ${ }^{[45]}$ 研究了二维层状 $\mathrm{Ti}_{3} \mathrm{C}_{2} \mathrm{~T}_{x} \mathrm{M}$ Xene纳米片在水介质中对 $\mathrm{Cu}(\mathrm{II})$ 的吸附和脱附过程. 与多层 $\mathrm{Ti}_{3} \mathrm{C}_{2} \mathrm{~T}_{x} \mathrm{MXene}$ (ML- $\mathrm{Ti}_{3} \mathrm{C}_{2} \mathrm{~T}_{x} \mathrm{MXene}$ )相比, 剥离 后的薄层 $\mathrm{Ti}_{3} \mathrm{C}_{2} \mathrm{~T}_{x}$ MXene (DL- $\mathrm{Ti}_{3} \mathrm{C}_{2} \mathrm{~T}_{x} \mathrm{MXene}$ ) 拥有更大 的比表面积、更好的亲水性以及更多的表面官能团, 展现了对 $\mathrm{Cu}(\mathrm{II})$ 更好的吸附性能. $\mathrm{DL}-\mathrm{Ti}_{3} \mathrm{C}_{2} \mathrm{~T}_{x}$ 表面的含

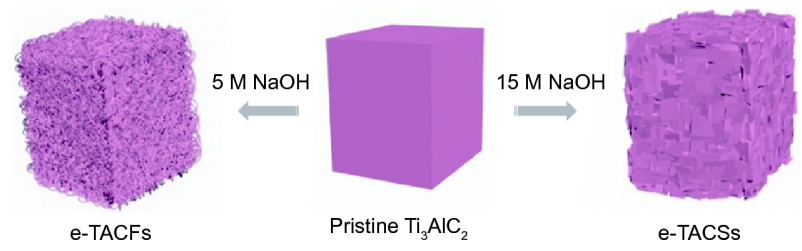

图 $5 \mathrm{Ti}_{3} \mathrm{AlC}_{2}$ (纳米片和纳米纤维)的制备示意图 ${ }^{[54]}$ (网络版 彩图)

Figure 5 Schematic of formation of etched $\mathrm{Ti}_{3} \mathrm{AlC}_{2}$ (nanosheets and nanofibers) [54] (color online).
氧官能团促进了 $\mathrm{Cu}(\mathrm{II})$ 的吸附还原. 实验结果显示, 最 大实验吸附容量高达 $78.5 \mathrm{mg} / \mathrm{g}, 80 \%$ 的 $\mathrm{Cu}(\mathrm{II})$ 在 $1 \mathrm{~min}$ 之内被吸附. 热力学分析显示吸附过程为吸热反应. 剥离后的薄层 $\mathrm{DL}-\mathrm{Ti}_{3} \mathrm{C}_{2} \mathrm{~T}_{x} \mathrm{MXene}$ 对 $\mathrm{Cu}(\mathrm{II})$ 的吸附量是 商用活性炭的2.7倍.

\subsubsection{Ba(II)的去除}

钡元素通常以化合物的形成存在，如 $\mathrm{BaSO}_{4}$ 和 $\mathrm{BaCO}_{3}$. 水体中一定浓度的钡可能会导致呼吸困难、 血压升高、胃部不适、脑肿胀、肌肉无力，损坏肝 脏、肾脏、心脏和脾脏, 还可能会引发癌症 ${ }^{[74,75]}$. Fard 等 ${ }^{[55]}$ 使用MXene材料 $\left(\mathrm{Ti}_{3} \mathrm{C}_{2} \mathrm{~T}_{x}\right)$ 去除水溶液中 $\mathrm{Ba}(\mathrm{II})$. 研 究结果显示, $\mathrm{Ti}_{3} \mathrm{C}_{2} \mathrm{~T}_{x} \mathrm{MXene}$ 对 $\mathrm{Ba}(\mathrm{II})$ 的吸附过程具有快 速的吸附动力学、较大的吸附容量和高选择性. 吸附 最佳 $\mathrm{pH}$ 为6 7. 在初始钡浓度为 $55 \mathrm{ppm}$ 时, 最大去除量 为 $9.3 \mathrm{mg} / \mathrm{g}$, 在优化条件下能达到 $100 \%$ 的去除效率, 吸 附性能优于活性炭和碳纳米管. 吸附最佳 $\mathrm{pH}$ 为 6 7. 碱 化后的MXene $\left(\mathrm{Alk}-\mathrm{Ti}_{3} \mathrm{C}_{2} \mathrm{~T}_{x}\right)$ 的吸附性能会显著提高. $\mathrm{Mu}$ 等 ${ }^{[73]}$ 研究发现, $\mathrm{Alk}-\mathrm{Ti}_{3} \mathrm{C}_{2} \mathrm{~T}_{x}$ 具有良好的层间距和丰 富的活性吸附位点，能够有效去除核废水中的放射性 $\mathrm{Ba}(\mathrm{II})$ (图6). Alk- $\mathrm{Ti}_{3} \mathrm{C}_{2} \mathrm{~T}_{x}$ 对 $\mathrm{Ba}(\mathrm{II})$ 的最大吸附容量为 $46.5 \mathrm{mg} / \mathrm{g}$, 是未碱化 $\mathrm{Ti}_{3} \mathrm{C}_{2} \mathrm{~T}_{x}$ 吸附容量的3倍.

\subsection{4 $\operatorname{Cr}(\mathrm{VI})$ 的去除}

+6 价铬是一种重金属元素, 是一种强大的氧化剂, 广泛应用于电镀、水冷、皮革鞣制和金属加工等工业 过程中 ${ }^{[76]}$. 这些工业的废水含有大量残留的 $\mathrm{Cr}(\mathrm{VI})$, 如

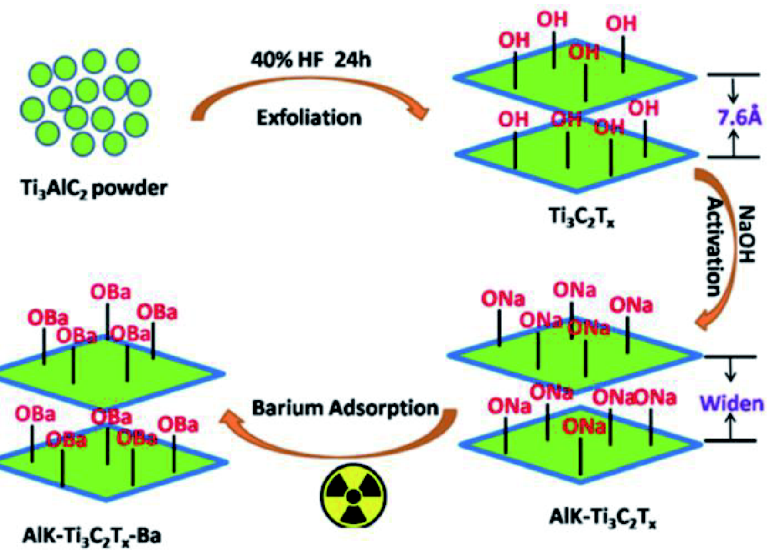

图 6 MXene材料吸附钡离子原理图 ${ }^{[73]}$ (网络版彩图)

Figure 6 Schematic diagram of the adsorption of barium ions [73] (color online). 
果没有经过适当无害化处理的排放, 会对地下水造成 严重的环境污染, 对植物、动物和人体都有剧毒 ${ }^{[77]}$.

Ying 等 ${ }^{[62]}$ 使用HF刻蚀法制备了 $\mathrm{Ti}_{3} \mathrm{C}_{2} \mathrm{~T}_{x}$ MXene, 并将其应用于 $\mathrm{Cr}(\mathrm{VI})$ 的吸附还原研究，发现 $\mathrm{Ti}_{3} \mathrm{C}_{2} \mathrm{~T}_{x}$ MXene对 $\mathrm{Cr}(\mathrm{VI})$ 有很好的吸附能力. 使用 $10 \% \mathrm{HF}$ 制备 的 $\mathrm{Ti}_{3} \mathrm{C}_{2} \mathrm{~T}_{x}$ MXene材料对 $\mathrm{Cr}(\mathrm{VI})$ 的吸附容量达到了 $250 \mathrm{mg} / \mathrm{g}$, 在 $\mathrm{pH} 5$ 时吸附效果最好, 吸附后溶液中残留 的 $\mathrm{Cr}(\mathrm{VI})$ 低于 $5 \mathrm{ppb}$ ，远低于世界卫生组织规定的饮用 水中浓度 $(0.05 \mathrm{ppm}) . \mathrm{Ti}_{3} \mathrm{C}_{2} \mathrm{~T}_{x} \mathrm{MXene}$ 纳米材料可以在 静电作用下快吸附 $\mathrm{Cr}(\mathrm{VI})$, 并将其一步还原为低毒性 的 $\mathrm{Cr}(\mathrm{III})$, 实现对 $\mathrm{Cr}(\mathrm{VI})$ 的高效吸附还原.

此外, Zou等 ${ }^{[56]}$ 使用自组装的合成方法, 在 $\mathrm{FeCl}_{3}$ 存 在的条件下, 通过MXene $\left(\mathrm{Ti}_{3} \mathrm{C}_{2}(\mathrm{OH})_{0.8} \mathrm{~F}_{1.2}\right)$ 的原位相变 制备了海胆状金红石 $\mathrm{TiO}_{2}-\mathrm{C}(\mathrm{u}-\mathrm{RTC})$ 纳米复合材料(图 7). $\mathrm{TiO}_{2}-\mathrm{C}(\mathrm{u}-\mathrm{RTC})$ 纳米复合碳材料在环境应用中表现 出优异的化学活性, 对 Cr(VI) 的吸附容量高达 $225 \mathrm{mg} / \mathrm{g}$, 远高于母体材料MXene ( $62 \mathrm{mg} / \mathrm{g})$. 第一性 原理的计算结果表明, $\mathrm{TiO}_{2}-\mathrm{C}$ (u-RTC)纳米复合碳材 料对 $\mathrm{Cr}(\mathrm{VI})$ 的高吸附量的原因是桥接的含氧基团抑制 了 $\mathrm{H}_{2} \mathrm{O}$ 分子的吸收. 该工作不仅找到了一种理想的 $\mathrm{Cr}(\mathrm{VI})$ 吸附材料, 还通过控制原位相变的方式对二维 材料进行自组装改性, 为进一步寻找MXene的其他衍 生物提供了新的方法.

\subsection{5 $\mathrm{Hg}(\mathrm{II})$ 的去除}

$\mathrm{Hg}(\mathrm{II})$ 是一种广泛存在的环境污染物. Shahzad 等 ${ }^{[57]}$ 通过水热合成法制备了磁性 $\mathrm{Ti}_{3} \mathrm{C}_{2} \mathrm{~T}_{x} \quad$ MXene复合 材料 $(\mathrm{MGMX})$, 用于去除水体中的有毒录离子. MGMX纳米复合材料在广泛的 $\mathrm{pH}$ 范围内(3.0 9.0), 表 现出优异的 $H g(I I)$ 去除性能, 对 $\mathrm{Hg}(\mathrm{II})$ 的最大实验吸收 容量高达 $1128.4 \mathrm{mg} / \mathrm{g}$. 在 $\mathrm{Mg}(\mathrm{II}) 、 \mathrm{Na}(\mathrm{I}) 、 \mathrm{Ca}(\mathrm{II})$ 和 $\mathrm{K}(\mathrm{I})$
干扰离子存在时，仍然有 $98 \%$ 的 $\mathrm{Hg}$ 吸附效果，展现了 高选择性. MGMX纳米复合材料在连续5次吸附-解吸 循环中重复使用，具有良好的吸附剂再生能力. MGMX纳米复合材料具有明显的高亲水性、高表面 积、水相稳定性、可循环性．这些因素表明，MGMX 纳米复合材料是一种实用的吸附剂, 可用于从废水处 理系统中去除致癌的Hg(II).

\section{5 总结与展望}

MXene材料在环境化学领域具有广阔的应用前 景. 本文系统地介绍了基于MXene材料的相关研究技 术, 如理论计算、批次实验和光谱技术. 总结了 MXene材料吸附剂对放射性核素(如U(VI))和有毒重 金属 $(\mathrm{Pb}(\mathrm{II}) 、 \mathrm{Cu}(\mathrm{II}) 、 \mathrm{Ba}(\mathrm{II}) 、 \mathrm{Cr}(\mathrm{VI}) 、 \mathrm{Hg}(\mathrm{II}))$ 等的吸 附行为. 相关实验研究和计算模拟结果表明, 将MXene 材料用于核素和重金属离子的去除具有吸附容量高、 动力学快、选择性好等优势. 未来MXene基材料在放 射性核素及有毒重金属离子富集分离领域仍有较大的 研究空间与发展潜力, 主要包括以下 3 个方面:

(1) 目前关于MXene与放射性元素的相互作用研 究工作仅涉及常见的 $\mathrm{Ti}_{3} \mathrm{C}_{2} \mathrm{~T}_{x} 、 \mathrm{~V}_{2} \mathrm{CT}_{x}$ 以及 $\mathrm{U}(\mathrm{VI})$, 尚未 扩展到其他类型的MXene以及放射性元素离子. 首先, 核燃料循环中关键的放射性元素 $(\mathrm{Am} 、 \mathrm{Pu} 、 \mathrm{~Np}$ 、 Tc、Sr、Cs等)是环境放射化学的重点研究对象, 开展 这些元素在环境中的常见种态与MXene的相互作用研 究非常迫切. 其次, 对于不同种类的MXene, 层结构与 成键性质的不同决定了它们物理化学性质的差异. 深 入研究不同种类MXene与目标离子的作用规律, 能够 衍生出MXene材料分离放射性元素以及重金属离子的 新技术和新原理. 例如, $\mathrm{Ti}_{2} \mathrm{CT}_{x}$ 相较于 $\mathrm{Ti}_{3} \mathrm{C}_{2} \mathrm{~T}_{x}$ 反应活性 更高，在高氧化态钶系离子的还原固定领域可能具有
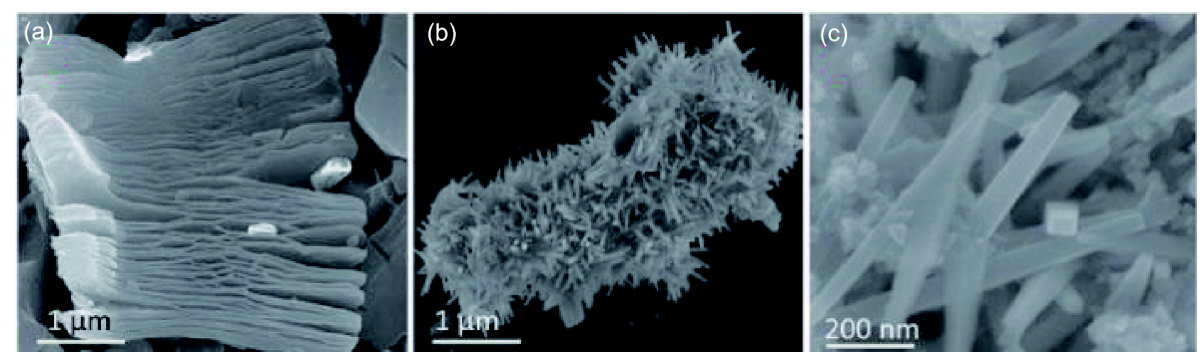

图 7 微观结构表征图 ${ }^{[56]}$. (a) 刻蚀后的MXene的SEM图; (b) u-RTC的SEM图; (c) (b)图的局部放大倍数图

Figure 7 Microstructural characterization [56]. (a) SEM image of Al-removed MXene; (b) SEM image of the u-RTC; (c) the local high magnification of (b). 
较好的应用前景.

(2) 进行MXene材料的功能化修饰是提高其吸附 性能的主要发展方向，未来可以进行高性能MXene复 合物、功能化、衍生物材料的制备并将其应用于放射 性元素与重金属离子的吸附分离. 同时利用MXene较 好的导电以及成膜特性，还可以开展其对放射性核素 及有毒重金属在电检测、电吸附、膜分离与固定化等
方面的研究.

（3）超薄MXene材料具有更大的比表面积和丰富 的活性位点，能够更好地实现放射性核素及有毒重金 属的富集和分离. 但单层MXene材料的产量低、不易 保存, 因此目前大多数吸附分离研究均使用了多层 MXene. 实现超薄MXene材料的宏量制备，以及提高 单层MXene的稳定性是该领域亟待解决的问题.

\section{参考文献}

1 Dincer I, Acar C. Int J Energy Res, 2015, 39: 585-606

2 Bundt M, Albrecht A, Froidevaux P, Blaser P, Flühler H. Environ Sci Technol, 2000, 34: 3895-3899

3 Fujita Y, Taylor JL, Gresham TLT, Delwiche ME, Colwell FS, McLing TL, Petzke LM, Smith RW. Environ Sci Technol, 2008, 42: 3025-3032

4 Latta DE, Mishra B, Cook RE, Kemner KM, Boyanov MI. Environ Sci Technol, 2014, 48: 1683-1691

5 Li J, Wang X, Zhao G, Chen C, Chai Z, Alsaedi A, Hayat T, Wang X. Chem Soc Rev, 2018, 47: 2322-2356

6 Sun Y, Yang S, Chen Y, Ding C, Cheng W, Wang X. Environ Sci Technol, 2015, 49: 4255-4262

7 Carboni M, Abney CW, Liu S, Lin W. Chem Sci, 2013, 4: 2396-2402

8 Yue Y, Mayes RT, Kim J, Fulvio PF, Sun XG, Tsouris C, Chen J, Brown S, Dai S. Angew Chem Int Ed, 2013, 52: 13458-13462

9 Camacho LM, Deng S, Parra RR. J Hazard Mater, 2010, 175: 393-398

10 Korichi S, Bensmaili A. J Hazard Mater, 2009, 169: 780-793

11 Lee SS, Li W, Kim C, Cho M, Lafferty BJ, Fortner JD. J Mater Chem A, 2015, 3: 21930-21939

12 Naguib M, Gogotsi Y. Acc Chem Res, 2015, 48: 128-135

13 Naguib M, Halim J, Lu J, Cook KM, Hultman L, Gogotsi Y, Barsoum MW. J Am Chem Soc, 2013, 135: 15966-15969

14 Naguib M, Mashtalir O, Carle J, Presser V, Lu J, Hultman L, Gogotsi Y, Barsoum MW. ACS Nano, 2012, 6: 1322-1331

Halim J, Lukatskaya MR, Cook KM, Lu J, Smith CR, Näslund LÅ, May SJ, Hultman L, Gogotsi Y, Eklund P, Barsoum MW. Chem Mater, 2014, 26: $2374-2381$ 1600050 

6227 9520 11391

Yang T, Wang C, Taylor CA, Huang X, Huang Q, Li F, Shen L, Zhou X, Xue J, Yan S, Wang Y. Acta Mater, 2014, 65: 351-359

Zhang H, Su R, Shi L, O’Connor DJ, King BV, Kisi EH. J Eur Ceramic Soc, 2018, 38: 1253-1264

Ghidiu M, Naguib M, Shi C, Mashtalir O, Pan LM, Zhang B, Yang J, Gogotsi Y, Billinge SJL, Barsoum MW. Chem Commun, 2014, 50: 9517-

Alhabeb M, Maleski K, Mathis TS, Sarycheva A, Hatter CB, Uzun S, Levitt A, Gogotsi Y. Angew Chem Int Ed, 2018, 57: 5444-5448

Urbankowski P, Anasori B, Makaryan T, Er D, Kota S, Walsh PL, Zhao M, Shenoy VB, Barsoum MW, Gogotsi Y. Nanoscale, 2016, 8: 11385-

Li T, Yao L, Liu Q, Gu J, Luo R, Li J, Yan X, Wang W, Liu P, Chen B, Zhang W, Abbas W, Naz R, Zhang D. Angew Chem, 2018, 130: 6223-

Zhou J, Zha X, Chen FY, Ye Q, Eklund P, Du S, Huang Q. Angew Chem, 2016, 128: 5092-5097

Zhou J, Zha X, Zhou X, Chen F, Gao G, Wang S, Shen C, Chen T, Zhi C, Eklund P, Du S, Xue J, Shi W, Chai Z, Huang Q. ACS Nano, 2017, 11: $3841-3850$

Mashtalir O, Cook KM, Mochalin VN, Crowe M, Barsoum MW, Gogotsi Y. J Mater Chem A, 2014, 2: 14334-14338

Kajiyama S, Szabova L, Iinuma H, Sugahara A, Gotoh K, Sodeyama K, Tateyama Y, Okubo M, Yamada A. Adv Energy Mater, 2017 , 7: 1601873

Ghidiu M, Halim J, Kota S, Bish D, Gogotsi Y, Barsoum MW. Chem Mater, 2016, 28: 3507-3514

Peng Q, Guo J, Zhang Q, Xiang J, Liu B, Zhou A, Liu R, Tian Y. J Am Chem Soc, 2014, 136: 4113-4116

Mashtalir O, Naguib M, Mochalin VN, Dall'Agnese Y, Heon M, Barsoum MW, Gogotsi Y. Nat Commun, 2013 , 4: 1716

Li L, Zhang M, Zhang X, Zhang Z. J Power Sources, 2017, 364: 234-241

Shahzad A, Rasool K, Miran W, Nawaz M, Jang J, Mahmoud KA, Lee DS. ACS Sustain Chem Eng, 2017, 5: 11481-11488

Guo J, Fu H, Zou G, Zhang Q, Zhang Z, Peng Q. J Alloys Compd, 2016, 684: 504-509

Guo X, Zhang X, Zhao S, Huang Q, Xue J. Phys Chem Chem Phys, 2016, 18: 228-233

Zhang YJ, Zhou ZJ, Lan JH, Ge CC, Chai ZF, Zhang P, Shi WQ. Appl Surf Sci, 2017, 426: 572-578

Berdiyorov GR, Madjet ME, Mahmoud KA. Appl Phys Lett, 2016, 108: 113110

Zhang YJ, Lan JH, Wang L, Wu QY, Wang CZ, Bo T, Chai ZF, Shi WQ. J Hazard Mater, 2016, 308: 402-410

Ladeira ACQ, Ciminelli VST, Duarte HA, Alves MCM, Ramos AY. Geochim Cosmochim Acta, 2001, 65: 1211-1217

Wang L, Tao W, Yuan L, Liu Z, Huang Q, Chai Z, Gibson JK, Shi W. Chem Commun, 2017, 53: 12084-12087

Wang L, Yuan L, Chen K, Zhang Y, Deng Q, Du S, Huang Q, Zheng L, Zhang J, Chai Z, Barsoum MW, Wang X, Shi W. ACS Appl Mater Interfaces, 2016, 8: 16396-16403

Gu P, Xing J, Wen T, Zhang R, Wang J, Zhao G, Hayat T, Ai Y, Lin Z, Wang X. Environ Sci-Nano, 2018, 5: 946-955

Fard AK, Mckay G, Chamoun R, Rhadfi T, Preud'Homme H, Atieh MA. Chem Eng J, 2017, 317: 331-342

Zou G, Guo J, Peng Q, Zhou A, Zhang Q, Liu B. J Mater Chem A, 2016, 4: 489-499

Shahzad A, Rasool K, Miran W, Nawaz M, Jang J, Mahmoud KA, Lee DS. J Hazard Mater, 2018, 344: 811-818

Jian M, Liu B, Zhang G, Liu R, Zhang X. Colloids Surfs A-Physicochem Eng Aspects, 2015, 465: 67-76

Aksoyoglu S. J Radioanal Nucl Chem, 1989, 134: 393-403

Saeed MM, Ahmed M, Chaudary MH, Gaffar A. Solvent Extr Ion Exch, 2003, 21: 881-898

Xu N, Hochella Jr MF, Brown Jr GE, Parks GA. Geochim Cosmochim Acta, 1996, 60: 2801-2815

Ying Y, Liu Y, Wang X, Mao Y, Cao W, Hu P, Peng X. ACS Appl Mater Interfaces, 2015, 7: 1795-1803

Santos JS, Teixeira LSG, dos Santos WNL, Lemos VA, Godoy JM, Ferreira SLC. Anal Chim Acta, 2010, 674: 143-156

Chen H, Huang S, Zhang Z, Liu Y, Wang X. Acta Chim Sin, 2017, 75: 560-574 (in Chinese) 陈海军, 黄舒怡, 张志宾, 刘云海, 王祥科. 化学学 报, 2017, 75: 560-574

65 Liang Y, Gu P, Yao W, Yu S, Wang J, Wang X. Prog Chem, 2017, 29: 1062-1071 (in Chinese) [梁宇, 顾鹏程, 姚文, 于淑君, 王建, 王祥科. 化学 进展, 2017, 29: 1062-1071]

Pang H, Wang X, Yao W, Yu S, Wang X. Sci Sin Chim, 2018, 48: 58-73 (in Chinese) 庞宏伟, 王祥学, 姚文, 于淑君, 王祥科. 中国科学: 化学, 2018, 1: 006

Yang S, Wang X, Chen Z, Li Q, Wei B, Wang X. Prog Chem, 2017, 30: 225-242 (in Chinese) [杨姗也, 王祥学, 陈中山, 李倩, 韦堑特, 王祥科. 化学进展, 2017, 30: 225-242] 
Luo BC, Yuan LY, Chai ZF, Shi WQ, Tang Q. J Radioanal Nucl Chem, 2016, 307: 269-276

Gao L, Yang Z, Shi K, Wang X, Guo Z, Wu W. J Radioanal Nucl Chem, 2010, 284: 519-526

Mu W, Du S, Yu Q, Li X, Wei H, Yang Y. Dalton Trans, 2018, 47: 8375-8381

Celebi O, Kilikli A, Erten HN. J Hazard Mater, 2009, 168: 695-703

Gingele F, Dahmke A. Paleoceanography, 1994, 9: 151-168

Owlad M, Aroua MK, Daud WAW, Baroutian S. Water Air Soil Pollut, 2009, 200: 59-77

Kimbrough DE, Cohen Y, Winer AM, Creelman L, Mabuni C. Critical Rev Environ Sci Tech, 1999, 29: 1-46

\title{
Research progress of MXene materials in radioactive element and heavy metal ion sequestration
}

\author{
Mao Fan ${ }^{1,2 \dagger}$, Lin Wang ${ }^{2 \dagger}$, Yujuan Zhang ${ }^{3}$, Chengxin $\mathrm{Pei}^{1}$, Zhifang Chai ${ }^{2}$, Weiqun $\mathrm{Shi}^{{ }^{2 *}}$ \\ ${ }^{1}$ State Key Laboratory of Nuclear, Biological and Chemical Protection for Civilian, Beijing 102205, China \\ ${ }^{2}$ Institute of High Energy Physics, Chinese Academy of Sciences, Beijing 100049, China \\ ${ }^{3}$ School of Materials Science and Engineering, University of Science and Technology Beijing, Beijing 100083, China \\ $\dagger$ These authors contributed equally to this work. \\ *Corresponding author (email: shiwq@ihep.ac.cn)
}

\begin{abstract}
Two-dimensional transition metal carbide/nitride (MXene) is a class of new layered nanomaterial discovered in 2011. Due to the novel structure and unique characteristics, MXene-based materials have been widely explored in the applications of energy storage and environmental remediation fields. MXene materials have the advantages of large specific surface areas, abundant active adsorption sites, good hydrophilicity and controllable layer space, and such purely inorganic materials also exhibit excellent radiation resistance and thermal conductivity, thus can be considered as ideal candidates for the sequestration of radioactive element and heavy metal ions. Herein we review the research progress in the controllable preparation of MXene materials and their removal performance for radionuclides and heavy metal ions in the past five years. The related experimental techniques, adsorption behaviors and interaction mechanisms have been systematically summarized. The prospective investigation trends and possible directions in this field are also proposed and discussed.
\end{abstract}

Keywords: MXene, radionuclide, heavy metal ion, adsorption, environmental remediation

doi: $10.1360 / \mathrm{N} 032018-00155$ 